\title{
P53 and Parkin co-regulate mitophagy in bone marrow mesenchymal stem cells to promote the repair of early steroid-induced osteonecrosis of the femoral head
}

\author{
Fei Zhang ${ }^{1,2}$, Wuxun Peng ${ }^{1,2}$, Jian Zhang ${ }^{1,2}$, Wentao Dong ${ }^{1,2}$, Jianhua $W^{1,2}$, Tao Wang ${ }^{2}$ and Zhihong Xie ${ }^{2}$
}

\begin{abstract}
Survival and stemness of bone marrow mesenchymal stem cells (BMSCs) in osteonecrotic areas are especially important in the treatment of early steroid-induced osteonecrosis of the femoral head (ONFH). We had previously used BMSCs to repair early steroid-induced ONFH, but the transplanted BMSCs underwent a great deal of stressinduced apoptosis and aging in the oxidative-stress (OS) microenvironment of the femoral-head necrotic area, which limited their efficacy. Our subsequent studies have shown that under OS, massive accumulation of damaged mitochondria in cells is an important factor leading to stress-induced apoptosis and senescence of BMSCs. The main reason for this accumulation is that OS leads to upregulation of protein 53 (P53), which inhibits mitochondrial translocation of Parkin and activation of Parkin's E3 ubiquitin ligase, which decreases the level of mitophagy and leads to failure of cells to effectively remove damaged mitochondria. However, P53 downregulation can effectively reverse this process. Therefore, we upregulated Parkin and downregulated P53 in BMSCs. We found that this significantly enhanced mitophagy in BMSCs, decreased the accumulation of damaged mitochondria in cells, effectively resisted stress-induced BMSCs apoptosis and senescence, and improved the effect of BMSCs transplantation on early steroidinduced ONFH.
\end{abstract}

\section{Introduction}

Effective treatment of early steroid-induced osteonecrosis of the femoral head (ONFH) is still a difficult and urgent problem in the field of orthopedics. Bone marrow mesenchymal stem cell (BMSCs) transplantation has been used to treat early steroid-induced $\mathrm{ONFH}^{1-3}$. Survival and stemness of BMSCs in the bone necrotic area are key to transplantation effectiveness ${ }^{4-7}$. After the femoral head suffers avascular necrosis, an oxidative-stress (OS) microenvironment forms in the osteonecrotic area ${ }^{8-10}$. The transplanted BMSCs undergo a great deal of stress-

\footnotetext{
Correspondence: Wuxun Peng (903463644@qq.com)

'Department of Orthopedics, The Affliated Hospital of Guizhou Medical

University, Guiyang, Guizhou 550004, China

${ }^{2}$ Guizhou Medical University, Guiyang, Guizhou 550004, China

Edited by G.M. Fimia
}

induced apoptosis and senescence in this microenvironment, which limits their efficacy ${ }^{11,12}$.

Central to OS is the release by impaired mitochondria of excessive reactive oxygen species (ROS) and apoptosisinducing factors, which, in turn, increases telomere consumption, arrests the cell cycle, activates signaling pathways such as protein 53 (P53) and protein 38 mitogen-activated protein kinase (P38MAPK), and leads to cellular-stress-induced apoptosis and senescence ${ }^{13-15}$. Mitophagy is the most important way to eliminate damaged mitochondria from cells ${ }^{16-18}$, it not only promotes mitochondrial renewal but also reduces excessive ROS and apoptosis-inducing factors released by the damaged mitochondria ${ }^{19}$. Therefore, normal mitophagy is essential for maintaining cell redox homeostasis and promoting cell survival under OS conditions. However,

\section{(c) The Author(s) 2020}

(c) (i) Open Access This article is licensed under a Creative Commons Attribution 4.0 International License, which permits use, sharing, adaptation, distribution and reproduction c. in any medium or format, as long as you give appropriate credit to the original author(s) and the source, provide a link to the Creative Commons license, and indicate if changes were made. The images or other third party material in this article are included in the article's Creative Commons license, unless indicated otherwise in a credit line to the material. If material is not included in the article's Creative Commons license and your intended use is not permitted by statutory regulation or exceeds the permitted use, you will need to obtain permission directly from the copyright holder. To view a copy of this license, visit http://creativecommons.org/licenses/by/4.0/. 
under such conditions the level of mitophagy is always decreased, causing massive accumulation of damaged mitochondria and leading to stress-induced apoptosis and senescence in cells ${ }^{20-22}$.

At present, the known mechanisms of mitophagy include the phosphatase and tensin homolog (PTEN)induced putative kinase protein 1 (PINK1)-Parkin, B-cell lymphoma 2 (Bcl2)/adenovirus $\mathrm{E} 1 \mathrm{~B} 19 \mathrm{kDa}$ proteininteracting protein 3 (BNIP3)-Nix, and FUN14 domaincontaining 1 (FUNDC1) pathways ${ }^{19}$. Studies have shown that under OS, mitophagy is mainly regulated by the PINK1-Parkin pathway ${ }^{23}$. When mitochondrial function is impaired, PINK1 aggregates in the extracorporeal membranes of damaged mitochondria and recruits Parkin there. Parkin is then phosphorylated to activate its E3 ubiquitin ligase activity and mediates ubiquitination of mitochondrial extracorporeal-membrane proteins, ultimately triggering mitophagy ${ }^{24}$.

It is well known that OS can lead to upregulation of $\mathrm{P}^{25-28}$. In addition, recent studies have shown that P53 can bind to Parkin's Really Interesting New Gene 0 (RING0) region, which interferes with Parkin's biological function, affecting mitochondrial quality control, biosynthesis, kinetic regulation, and cellular redox homeostasis $^{19,29-31}$. Parkin's main function in mitochondrial quality control is to label damaged mitochondria via ubiquitin ligase activity, which, in turn, mediates mitophagy. Studies in mouse cardiomyocytes have shown that the binding of P53 to Parkin inhibits Parkin's E3 ubiquitin ligase activity ${ }^{29}$. However, the significance of this process in BMSCs remains to be determined, and it is unclear whether it might interfere with mitophagy mediated by PINK1-Parkin. It is also not yet clear whether downregulating P53 expression while upregulating that of Parkin could significantly enhance mitophagy in order to resist stress-induced apoptosis and senescence of BMSCs, thereby improving the effectiveness of BMSCs transplantation.

In this study, we preliminarily explored the roles and mechanisms of P53 and Parkin in regulating mitophagy, as well as the effect of enhanced mitophagy on stressinduced apoptosis and senescence of BMSCs. We further evaluated the effect of P53-Parkin co-regulation of mitophagy on the repair of early steroid-induced ONFH by BMSCs transplantation.

\section{Results}

Repair of early steroid-induced ONFH in rabbits via BMSCs transplantation

BMSCs have strong self-renewal ability and multidirectional differentiation potential ${ }^{32-34}$. In order to study their osteogenic potential, we induced osteogenic differentiation of BMSCs. After 2 weeks, a large number of calcium nodules were formed, alizarin red and alkaline phosphatase (ALP) staining results were positive, and we confirmed that the BMSCs had strong osteogenic potential in vitro (Fig. S1). To observe BMSCs osteogenesis in vivo, we used lipopolysaccharide (LPS) combined with methylprednisolone to establish an early steroid-induced ONFH model in rabbits. At week 8, magnetic resonance imaging (MRI; T2-weighted image [WI]) showed mixed signals of different heights in the femoral head (Fig. 1a). Hematoxylin and eosin (H\&E) staining showed that the medullary cavity was filled with a large amount of adipose tissue, the trabecular bone had become thinner, and no osteoblast-related cells were present (Fig. 1b). These results confirmed the success of our early steroid-induced ONFH model.

Subsequently, we inoculated BMSCs into xenogeneic antigen-extracted cancellous bone (XACB) for co-culture to create tissue-engineered bone (BMSCs/XACB). On day 6 , we observed BMSCs growing on the surface of the XACB using a scanning electron microscope (SEM); these BMSCs had good biocompatibility (Fig. 1c). Finally, we transplanted the BMSCs/XACB into the rabbits to repair early steroid-induced ONFH (Fig. 1d). At 12 weeks postsurgery, we evaluated the repair of the femoral-head necrotic area by H\&E staining, Masson staining, and detection of osteogenic markers such as osteoprotegerin (OPG), osteocalcin (OCN), and runt-related transcription factor 2 (Runx2). The results showed that only a small amount of new-bone tissue had formed in the necrotic area of the XACB group and the BMSCs/XACB group, and no mature bone tissue was found (Fig. 1e). Also, there were no significant difference in the area of new-bone formation and in levels of osteogenic markers (Fig. 1f-i). In conclusion, after tissue-engineered bone implantation into the femoral-head necrotic area, there was no significant difference in osteogenesis between the BMSCs/ $\mathrm{XACB}$ and $\mathrm{XACB}$ groups. Therefore, we had reason to speculate that there might be important factors affecting the survival and osteogenic differentiation of BMSCs in necrosis of the femoral head.

\section{Excessive accumulation of damaged mitochondria led to stress-induced apoptosis and senescence of BMSCs}

In the avascular necrotic area of the femoral head, the function of the oxygenated mitochondrial respiratory chain is impaired by ischemia and hypoxia, producing excessive ROS. Moreover, after ischemic necrosis of the femoral head, inflammatory cells infiltrate, and the released inflammatory mediators also mediate ROS production by the inflammatory cells. Therefore, after necrosis of the femoral head, a local OS microenvironment forms ${ }^{8-11}$. In order to further study the effects of OS on BMSCs and then to treat the BMSCs, we used high-concentration $\mathrm{H}_{2} \mathrm{O}_{2}(1000 \mu \mathrm{M})$ to simulate an OS microenvironment ${ }^{35}$. We used $\mathrm{JC}-1$ to 
A

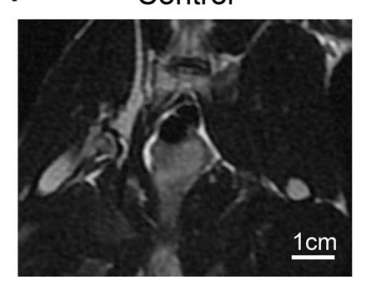

C

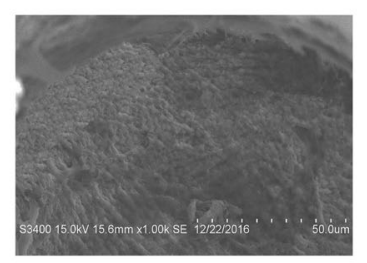

$\mathrm{E}$
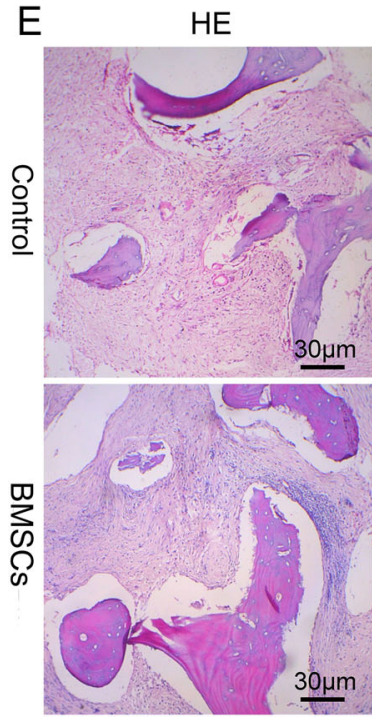

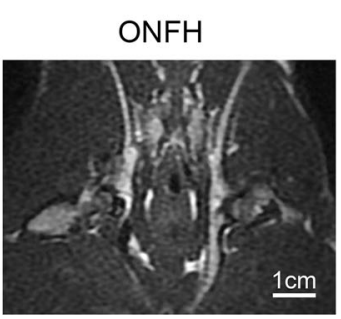

XACB/BMSCs
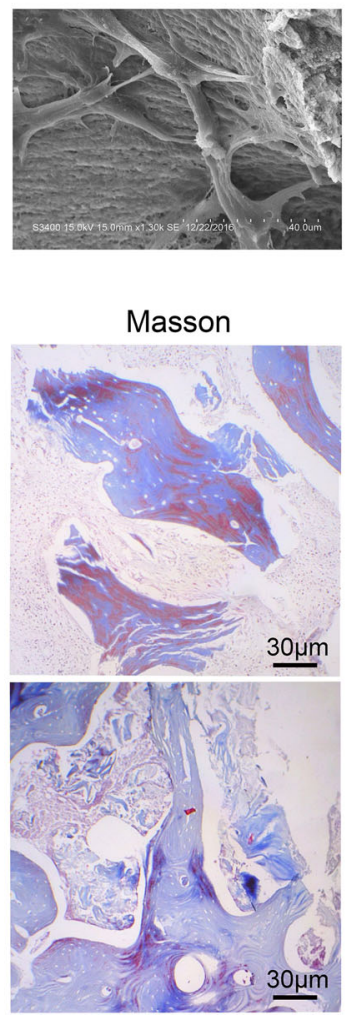

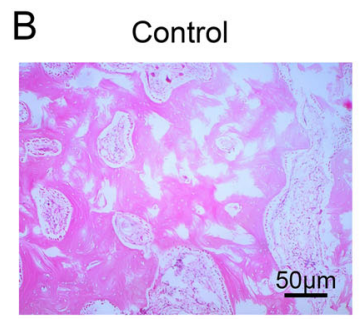

D
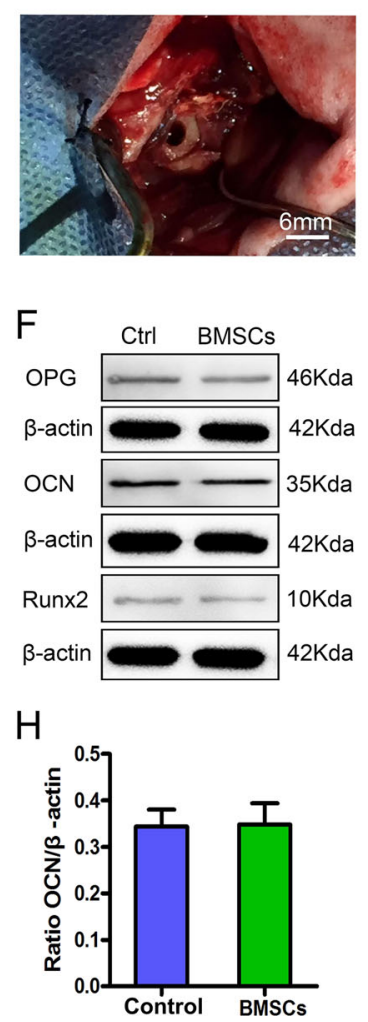

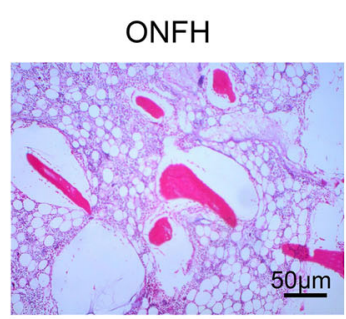

Transplantation

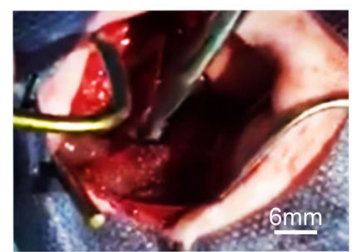

G
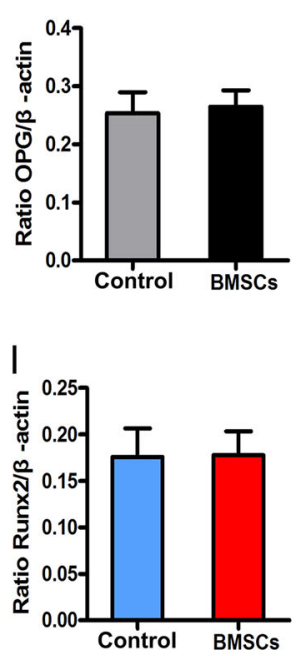

Fig. 1 Osteogenesis of BMSCs in vivo. a Magnetic resonance imaging (MRI) examination of the ONFH model. $\mathbf{b}$ H\&E staining of the ONFH model. Lipopolysaccharide (LPS) and methylprednisolone were used to build the model; saline was used as control. c Observation of XACB and XACB/BMSCS via scanning electron microscopy (SEM). d BMSCs repair of ONFH. Control was treated with lesion debridement (LD) without BMSCs implantation. e H\&E and Masson staining to detect new-bone formation and maturation. $\mathbf{f}-\mathbf{i}$ Western blot analysis of OPG, OCN, and Runx2 expression in osteonecrosis $(n=3)$. In $(\mathbf{g}-\mathbf{i})$, data are presented as means \pm standard deviation (SD). Statistical significances were calculated by Student's $t$ test. BMSCs = bone marrow mesenchymal stem cells, ONFH = osteonecrosis of the femoral head, XACB = xenogeneic antigen-extracted cancellous bone, $\mathrm{XACB} / \mathrm{BMSCs}=$ tissue-engineered bone, $\mathrm{OPG}=$ osteoprotegerin, $\mathrm{OCN}=$ osteocalcin, Runx2 = runt-related transcription factor 2.

measure mitochondrial-membrane potential (MMP), and MitoTracker Green staining and a quantitative mitochondrial deoxyribonucleic acid (mtDNA) assay to assess intracellular mitochondrial content. The results showed that after BMSCs were subjected to OS, MMP decreased, mitochondrial function was impaired (Fig. 2a, b), and the damaged-mitochondria content increased significantly in cells (Fig. 2c-e).

Subsequently, we used a $2^{\prime}, 7^{\prime}$-dichlorodihydrofluorescein diacetate (DCFH-DA) fluorescent probe to detect ROS levels, terminal deoxynucleotidyl transferase deoxyuridine- $5^{\prime}$-triphosphate nick end labeling (TUNEL) staining to detect DNA damage, Annexin V-FITC and propidium iodide (PI) staining to detect apoptosis, and $\beta$-galactosidase ( $\beta$-gal) staining and examination of protein 21 (P21) and other senescence-associated protein levels to assess cell senescence. The results showed that with the increase of intracellular damaged-mitochondria content, intracellular ROS levels also increased significantly (Fig. 3a, b), and DNA damage increased as well (Fig. 3c, d). Eventually, a large number of BMSCs were induced to undergo apoptosis (Fig. 3e, f), $\beta$-gal activity 


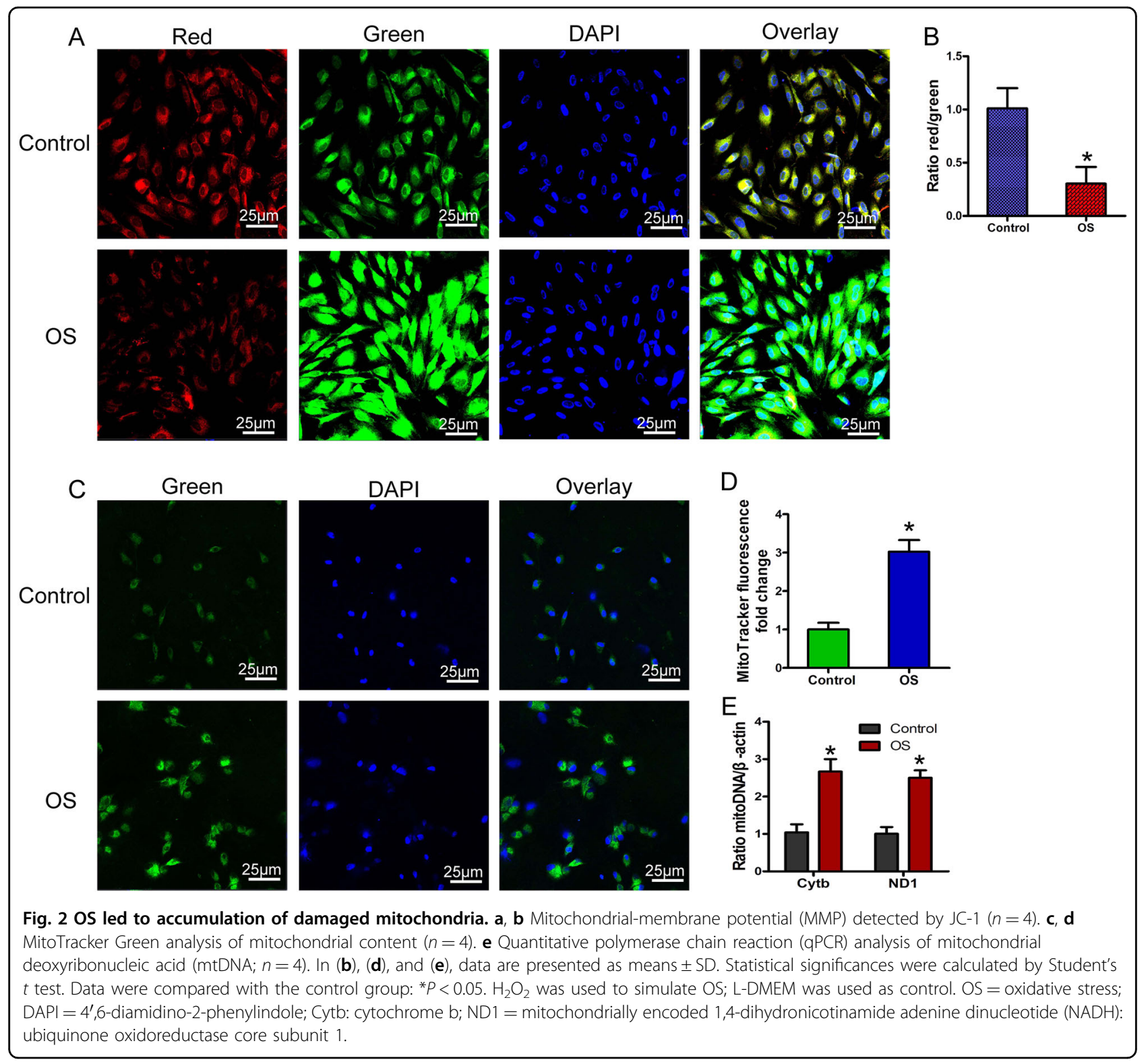

increased in the surviving BMSCs (Fig. 3g, h), the expression of aging-related proteins such as P21 and protein 16 (P16) was also significantly increased (Fig. $3 \mathrm{i}-\mathrm{k})$, and the surviving BMSCs showed stress-induced aging. Therefore, our results showed that stress-induced apoptosis and aging of BMSCs were not only related to mitochondrial dysfunction but also related to accumulation of damaged mitochondria in cells.

\section{Enhancing mitophagy against BMSCs stress-induced apoptosis and senescence}

Stress-induced apoptosis and senescence in BMSCs are related to the accumulation of damaged mitochondria in cells $^{20-22,36}$. Could enhancing mitophagy to clear these accumulated mitochondria effectively resist BMSCs stress-induced apoptosis and senescence? Mitophagy mediated by the PINK1-Parkin pathway is the most important way to clear damaged mitochondria ${ }^{37,38}$; mitochondrial translocation of Parkin is very important to mitophagy $^{39}$, and therefore increasing the content of Parkin in cells can be expected to increase mitochondrial translocation of Parkin and thus enhance mitophagy. Therefore, we used lentiviral vector-encoded Parkinenhanced green fluorescent protein (Lv-Parkin-EGFP) to transfect BMSCs. The results of quantitative polymerase chain reaction (qPCR) and western blot confirmed that Parkin was successfully overexpressed in BMSCs (Fig. 4a-d). We then used high-concentration $\mathrm{H}_{2} \mathrm{O}_{2}$ $(1000 \mu \mathrm{M})$ to simulate OS in order to treat BMSCs for $24 \mathrm{~h}^{35}$, hoping that we could enhance mitophagy by 


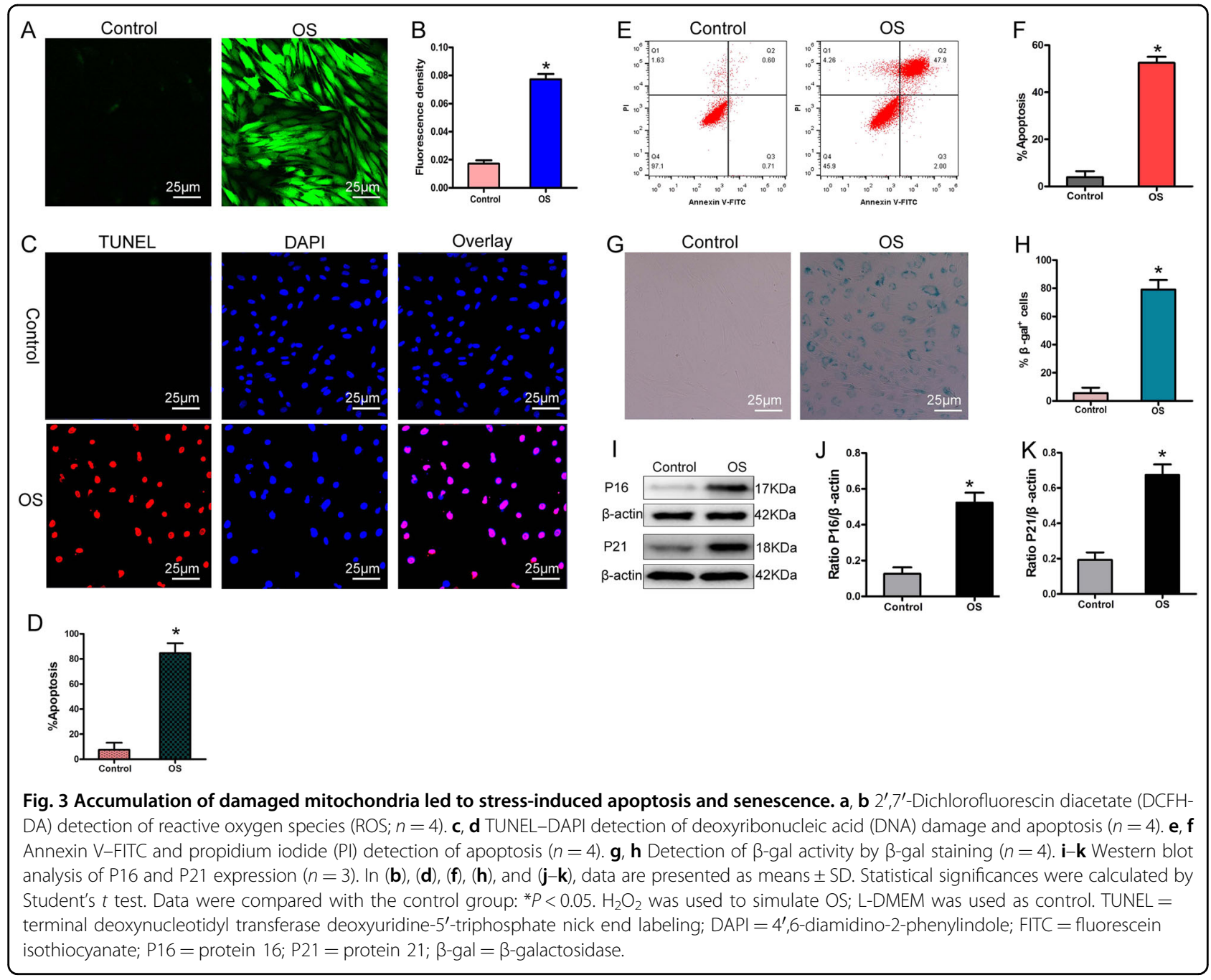

interfering with the PINK1-Parkin pathway. However, our immunocoprecipitation and western blot results showed that under OS, mitochondrial translocation of Parkin and the ubiquitination level of voltage-dependent anion-selective channel 1 (VDAC1) were increased slightly after lentivirus-mediated overexpression of Parkin (Fig. 4e-f). Electron microscopy showed that the number of mitophagic bodies in the BMSCs also increased slightly (Fig. 4g), but levels of damaged mitochondria (Fig. 4h, $\mathrm{k}-\mathrm{l}$, apoptosis, and $\beta$-gal activity in Parkinoverexpressing BMSCs were not significantly decreased compared with the control group (Fig. $4 \mathbf{i}-\mathbf{j}, \mathrm{m}-\mathrm{n}$ ). In conclusion, upregulation of Parkin expression alone had limited effects on enhancing mitophagy and resisting stress-induced apoptosis and aging in BMSCs. However, it is interesting to note that expression of P53 in cells was significantly upregulated after OS was induced in each group of BMSCs (Fig. 4o-q). Recent studies have shown that P53 can bind to Parkin and interfere with its physiological functions ${ }^{29-31}$. This may interfere with Parkin- mediated mitophagy, limiting the ability of Parkin upregulation alone to enhance mitophagy.

When we further downregulated P53 expression via the short-hairpin ribonucleic acid (shRNA) technique in the Parkin-BMSCs that we had established in the early stage, qPCR and western blot results confirmed that the BMSCs overexpressed Parkin, while P53 expression was significantly downregulated (Fig. 5a-f). We also treated BMSCs with $1000 \mu \mathrm{M} \mathrm{H}_{2} \mathrm{O}_{2}$ for $24 \mathrm{~h}$, which caused them to suffer from OS. The results showed that upregulation of Parkin and downregulation of P53 could further increase the number of mitophagic bodies in BMSCs on the basis of Parkin upregulation alone (Fig. 5g), and MitoTracker Green staining showed that damagedmitochondria content in the cells also decreased (Fig. 5h, k-m). We further evaluated stress-induced apoptosis and senescence of BMSCs by quantitative detection of Annexin V-FITC/PI, $\beta$-gal staining, and aging-related proteins. The results showed that under OS, Parkin upregulation and P53 downregulation in BMSCs 


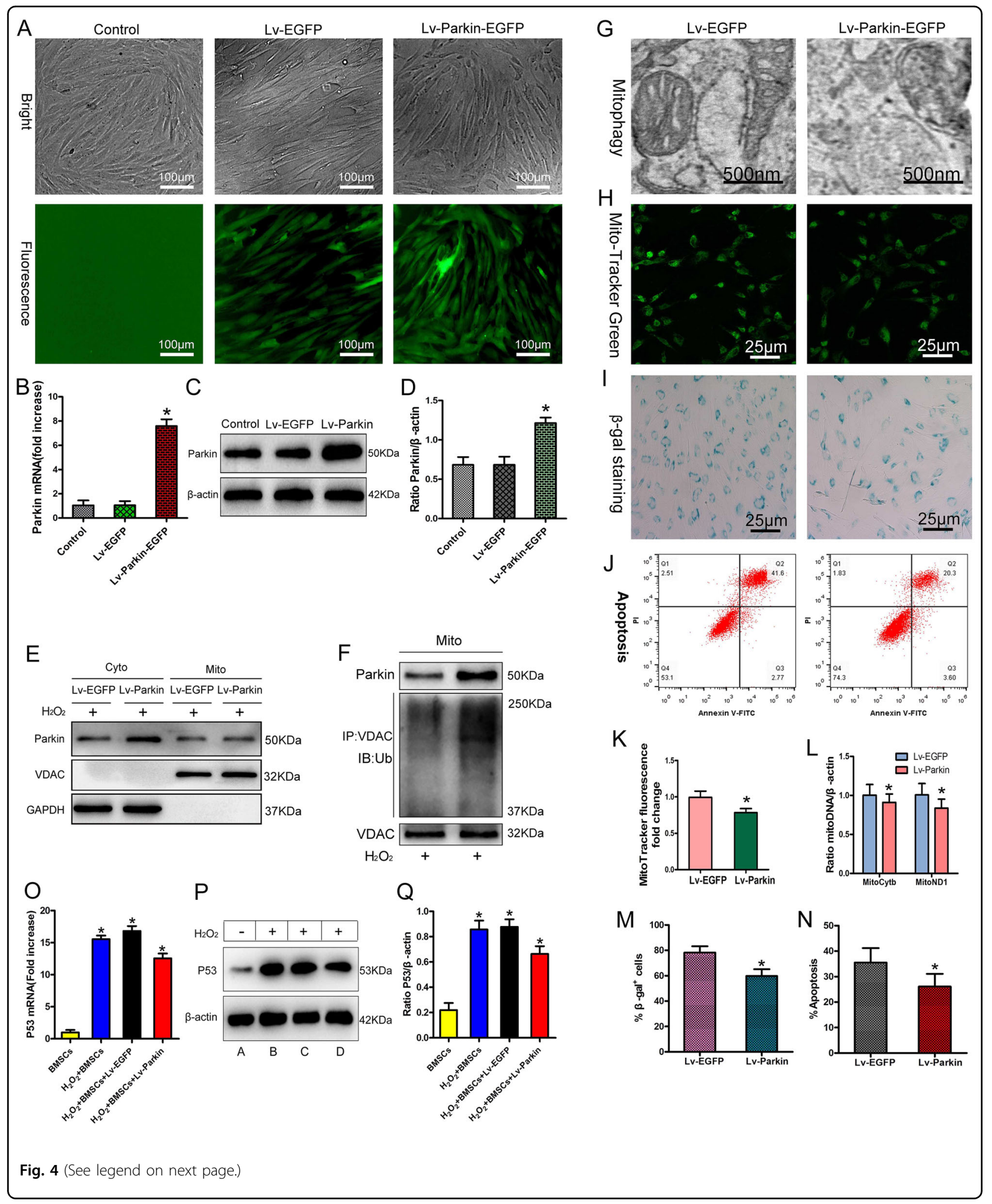




\begin{abstract}
(see figure on previous page)
Fig. 4 Enhancing mitophagy against stress-induced apoptosis and senescence in BMSCs. a Observation of fluorescent protein by inverted fluorescence microscopy $(n=4)$. $\mathbf{b}$ qPCR analysis of Parkin mRNA expression $(n=4)$. $\mathbf{c}$, d Western blot analysis of Parkin expression $(n=4)$. Nontransfected BMSCs as control. e Western blot analysis of Parkin mitochondrial translocation $(n=3)$. $\mathbf{f}$ Immunocoprecipitation (IP) and immunoblot (IB) analysis of VDAC1 ubiquitination level $(n=3)$. $\mathbf{g}$ Observation of mitophagy by transmission electron microscopy (TEM; $n=4)$. $\mathbf{h}, \mathbf{k}$ MitoTracker Green analysis of mitochondrial content $(n=4)$. i, $\mathbf{m}$ Detection of $\beta$-gal activity by $\beta$-gal staining $(n=4)$. $\mathbf{j}, \mathbf{n}$ Annexin $V$-FITC and PI detection of apoptosis $(n=4)$. I qPCR analysis of mitochondrial deoxyribonucleic acid $(m t D N A ; n=4)$. o qPCR analysis of P53 mRNA expression $(n=4)$. $\mathbf{p}, \mathbf{q}$ Western blot analysis of P53 expression $(n=4)$. In (p), A = BMSCs, B $=\mathrm{H}_{2} \mathrm{O}_{2}+\mathrm{BMSC}, \mathrm{C}=\mathrm{H}_{2} \mathrm{O}_{2}+\mathrm{BMSCs}+\mathrm{Lv}-\mathrm{EGFP}, \mathrm{D}=\mathrm{H}_{2} \mathrm{O}_{2}+\mathrm{BMSC}+\mathrm{Lv}$-Parkin. In (b), (d), (k$\mathbf{0})$, and $(\mathbf{q})$, data are presented as means \pm SD. Statistical significances were calculated by ANOVA and Student's $t$ test. In (b) and (d), data were compared with the control and Lv-EGFP groups separately; vs. control and Lv-EGFP: ${ }^{P} P<0.05$. In (k-n), data were compared with the Lv-EGFP group: ${ }^{*} P<0.05$. In (o) and (q), data were compared with the BMSCs group: ${ }^{*} P<0.05$. Lv-EGFP $=$ lentiviral vector-encoded green fluorescent protein; $L v-$ Parkin-EGFP = lentiviral vector-encoded Parkin-enhanced green fluorescent protein; $\mathrm{mRNA}=$ messenger ribonucleic acid; $\mathrm{Ub}=\mathrm{ubiquitin} ; \mathrm{VDAC1}=$ voltage-dependent anion-selective channel 1; P53 = protein 53; GAPDH = glyceraldehyde 3-phosphate dehydrogenase.
\end{abstract}

significantly decreased the apoptosis rate (Fig. 5i, n), and senescence phenotypes such as $\beta$-gal activity also decreased significantly (Fig. 5j, o). This evidence suggested that upregulating Parkin and downregulating P53 expression could significantly enhance mitophagy, thereby removing damaged mitochondria from cells and enabling BMSCs to effectively resist stress apoptosis and aging. It also showed that under OS, P53 also participated in regulation of mitophagy.

\section{Mechanism of P53 regulating mitophagy}

OS can upregulate the expression of P53 in cells ${ }^{40,41}$. Some studies have shown that P53 can interact with Parkin's RING0 region ${ }^{30,31}$. The PINK1-Parkin pathway is the most important pathway by which mitophagy is mediated $^{42}$; therefore, to further study whether P53 regulated mitophagy via this pathway, we continued to treat BMSCs for $24 \mathrm{~h}$ using an $\mathrm{H}_{2} \mathrm{O}_{2}(1000 \mu \mathrm{M})$-simulated OS. The results of qPCR and western blot confirmed that P53 expression was upregulated in the $\mathrm{H}_{2} \mathrm{O}_{2}{ }^{+}$EGFP and $\mathrm{H}_{2} \mathrm{O}_{2}{ }^{+}$Parkin groups, compared with the $\mathrm{H}_{2} \mathrm{O}_{2}{ }^{-}$EGFP and $\mathrm{H}_{2} \mathrm{O}_{2}{ }^{-}$Parkin groups. Subsequently, our immunocoprecipitation and immunoblot results showed that P53 upregulation increased the interaction between P53 and Parkin, while decreasing mitochondrial translocation of Parkin and the ubiquitination level of VDAC1 (Fig. 6a). Transmission electron microscopy (TEM) showed that the number of mitophagic bodies decreased (Fig. 6b); MitoTracker Green staining and mtDNA quantification showed that damaged-mitochondria content in cells was significantly increased (Fig. 6c-f). After we downregulated P53 expression of in BMSCs using shRNA, the interaction between P53 and Parkin in the $\mathrm{H}_{2} \mathrm{O}_{2}{ }^{+}$shP53 and $\mathrm{H}_{2} \mathrm{O}_{2}{ }^{+}$ Parkin/shP53 groups was reduced, compared with the $\mathrm{H}_{2} \mathrm{O}_{2}{ }^{+}$EGFP and $\mathrm{H}_{2} \mathrm{O}_{2}{ }^{+}$Parkin groups. In addition, mitochondrial translocation of Parkin and the ubiquitin level of VDAC1 increased, the level of mitophagy increased, and the content of damaged mitochondria in cells was significantly decreased (Fig. 6a-f). At the same time, we could see that upregulation of Parkin and downregulation of P53 significantly increased Parkin mitochondrial translocation and ubiquitination of mitochondrial extracorporeal-membrane proteins, as well as significantly enhancing mitophagy (Fig. 6a-f). These results suggested that P53 could regulate mitophagy via the PINK1-Parkin pathway.

\section{Parkin-shP53 BMSCs repaired early steroid-induced ONFH}

In the OS microenvironment that forms in the avascular necrotic area of the femoral head, accumulation of damaged mitochondria causes apoptosis and senescence of transplanted BMSCs, which seriously affects the transplantation effect. Our results showed that upregulation of Parkin expression and downregulation of P53 expression could significantly enhance mitophagy and then clear damaged mitochondria from cells, which could help BMSCs effectively resist stress apoptosis and aging (Fig. 5). To further evaluate whether enhanced mitophagy could further improve the reparative effect of BMSCs on early steroid-induced $\mathrm{ONFH}$, we first co-transfected BMSCs with Lv-Parkin and Lv-shP53 so that the BMSCs would upregulate Parkin and downregulate P53 simultaneously. Then, we co-cultured these BMSCs with $\mathrm{XACB}$ to construct tissue-engineered bone, which we used to repair early steroid-induced ONFH model in rabbits. At 12 weeks postsurgery, observation of gross specimens showed that the defect area in the XACB/ BMSCs/Parkin and $\mathrm{XACB} / \mathrm{BMSC} / \mathrm{sh} 553$ groups were significantly smaller than in the control, $\mathrm{XACB}$, and $\mathrm{XACB} / \mathrm{BMSCs}$ groups, but in all five of these groups the defect area was not completely repaired. However, in the $\mathrm{XACB} / \mathrm{BMSCs} /$ Parkin/shP53 group, the area was completely repaired (Fig. 7a).

Next, we evaluated new-bone formation by H\&E staining (Fig. 7c) and new-bone maturation by Masson staining (Fig. 7b). Total protein was extracted from femoral-head tissue, and levels of osteogenic markers such as Runx2, OCN, and OPG were detected by 


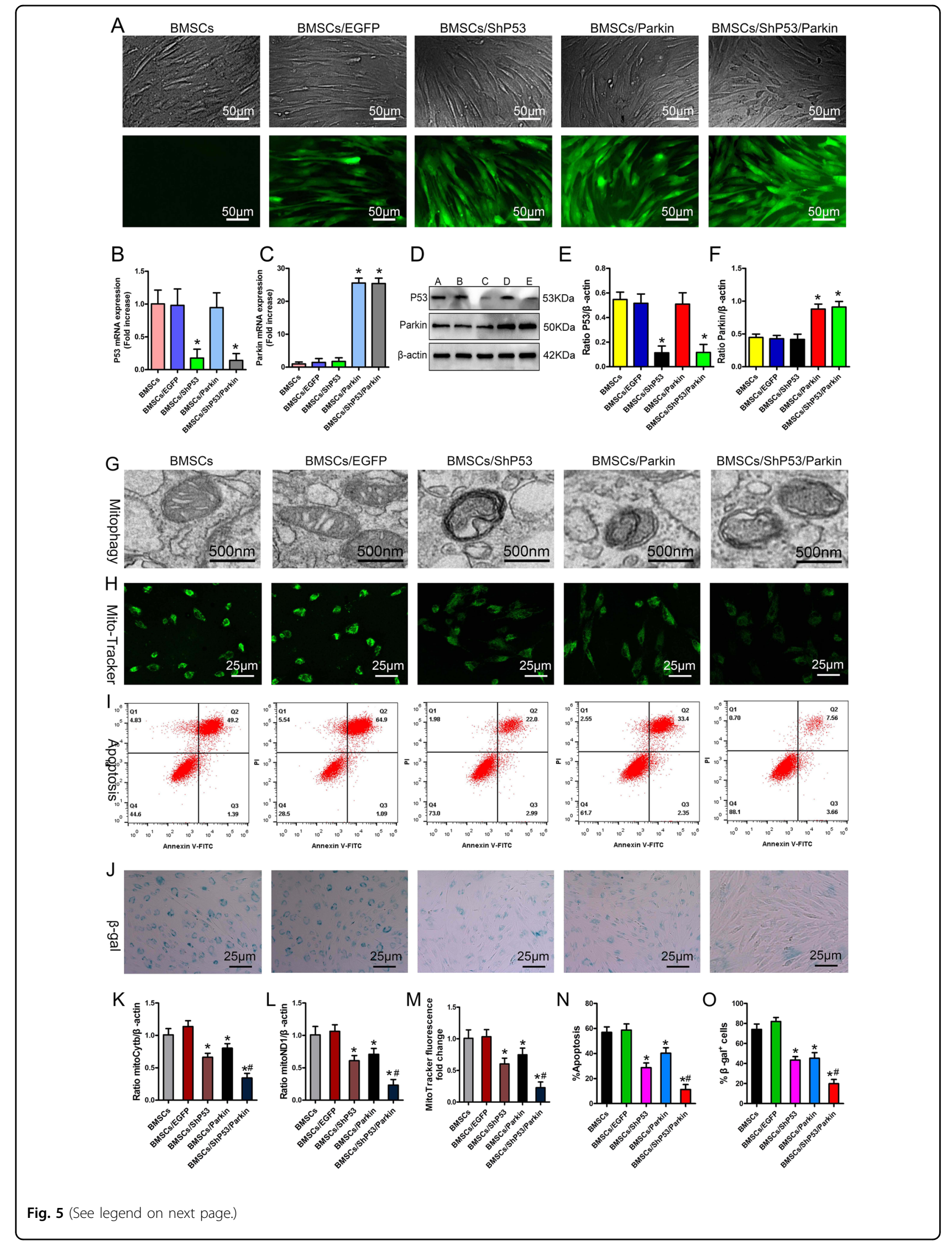


(see figure on previous page)

Fig. 5 Effects of mitophagy co-regulated by P53 and Parkin on stress-induced apoptosis and aging. a Observation of fluorescent protein by inverted fluorescence microscopy $(n=4)$. b , c qPCR analysis of P53 and Parkin mRNA expression $(n=4)$. $\mathbf{d}-\mathbf{f}$ Western blot analysis of P53 and Parkin expression $(n=4)$. $\mathbf{g}$ Observation of mitophagy by TEM $(n=3) . \mathbf{h}, \mathbf{m}$ MitoTracker Green analysis of mitochondrial content $(n=3)$. $\mathbf{i}, \mathbf{n}$ Annexin V-FITC and PI detection of apoptosis $(n=3)$. $\mathbf{j}$, o Detection of $\beta$-gal activity by $\beta$-gal staining $(n=3)$. $\mathbf{k}, \mathbf{I}$ gPCR analysis of $\operatorname{mtDNA}(n=3)$. In $(\mathbf{d})$, A $=$ BMSCs,

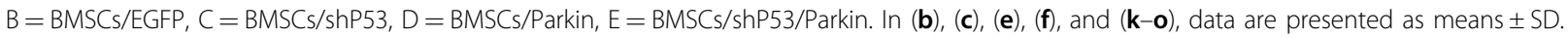
Statistical significances were calculated by ANOVA. In $(\mathbf{b}, \mathbf{c})$ and $(\mathbf{e}, \mathbf{f})$, data were compared with the BMSCs and BMSCs/EGFP groups: ${ }^{*} P<0.05$. In $(\mathbf{k}-\mathbf{o})$, data were compared with the BMSCs and BMSCs/EGFP groups: ${ }^{*} P<0.05$; or with the BMSCs/shP53 and BMSCs/Parkin groups: ${ }^{*} P<0.05$. shP53 $=$ protein 53 short-hairpin ribonucleic acid.

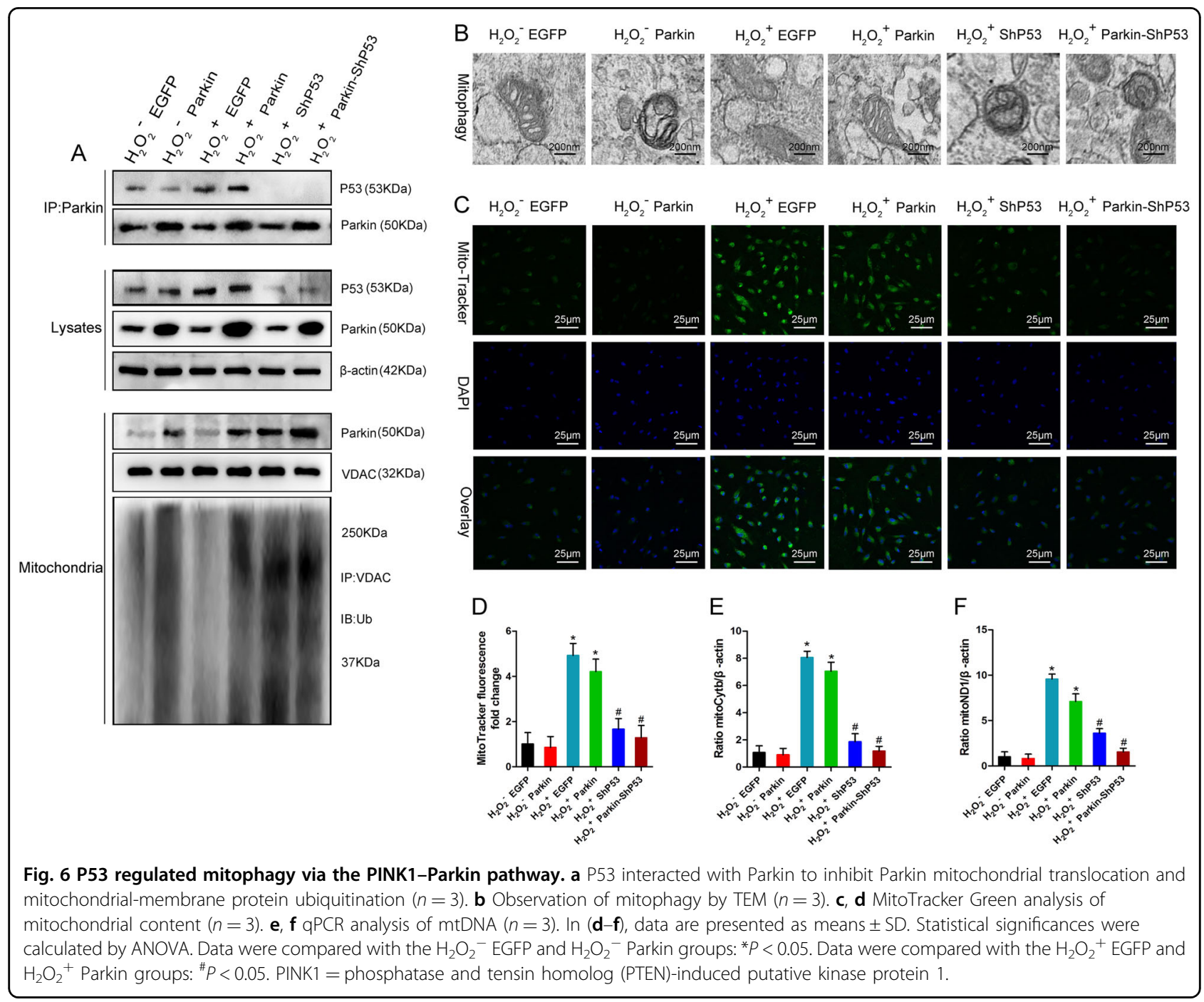

western blot (Fig. $7 \mathrm{~d}-\mathrm{g})$. The results showed that newbone formation and osteogenic-marker levels in the $\mathrm{XACB} / \mathrm{BMSC}$ /Parkin, XACB/BMSCs/shP53, and $\mathrm{XACB} / \mathrm{BMSCs} /$ Parkin/shP53 groups were higher than in the control, $\mathrm{XACB}$, and $\mathrm{XACB} / \mathrm{BMSCs}$ groups. Compared with the other groups, however, both metrics were highest in the $\mathrm{XACB} / \mathrm{BMSC} /$ Parkin/
shP53 group, the new-bone tissue had completely matured, and the boundary between new and normal bone was not clear (Fig. 7b-g). These results suggested that upregulation of Parkin and downregulation of P53 to enhance mitophagy of BMSCs could effectively improve the reparative effect of BMSCs on early steroid-induced ONFH. 


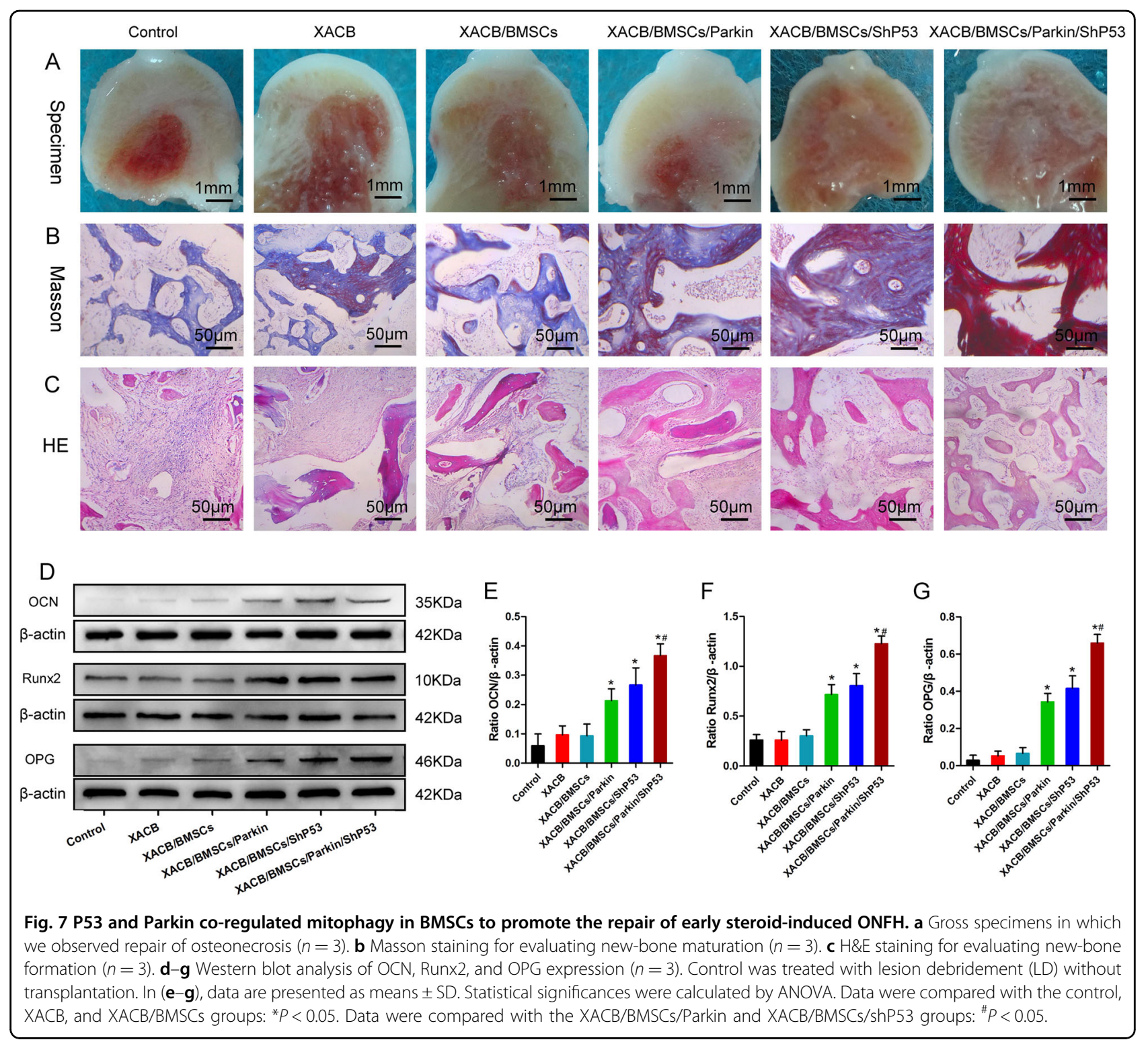

\section{Discussion}

BMSCs are used to treat early steroid-induced $\mathrm{ONFH}^{43-45}$; their survival and stemness in the bone necrotic area are key to the effectiveness of transplantation $^{46,47}$. Studies have revealed an OS microenvironment in the femoral-head necrotic area in which transplanted BMSCs incur a great deal of stress apoptosis and aging, which seriously limits transplantation effectiveness ${ }^{11-13}$. More and more studies have shown that mitochondrial dysfunction and damaged-mitochondria accumulation occur before cell stress apoptosis and senescence ${ }^{19-22}$. In this study, we preliminarily confirmed that the accumulation of damaged mitochondria was an important cause of BMSCs stress apoptosis and aging. Clearance of these mitochondria mainly depends on mitophagy mediated by the PINK1-Parkin pathway ${ }^{48}$. Our further experiments confirmed that under OS, the decrease in mitophagy mediated by this pathway could lead to accumulation of damaged mitochondria. OS led to upregulation of P53 expression; P53 interacted with Parkin to inhibit Parkin's mitochondrial translocation and further activation of E3 ubiquitin ligase, which in turn reduced VDAC1 ubiquitination and mitophagy levels. Clearance of damaged mitochondria was obstructed, which eventually led to their accumulating in cells. By using the shRNA technique to downregulate P53 expression, we could effectively reverse the above process. However, in this study we did not further investigate the mechanism of P53 upregulation induced by OS or the interaction site between P53 and Parkin. Related studies in cardiomyocytes and neurons indicate that OS increases P53 expression mainly by initiating the DNA damage response (DDR) and 
mammalian target of rapamycin (mTOR) pathways ${ }^{26,27}$. After its expression is upregulated, P53 can bind to the RING0 region of Parkin and then interfere with Parkin's biological function ${ }^{30,31}$. Whether this is also true in BMSCs requires further study.

Under OS, mitochondria suffer functional damage and accumulate in cells, releasing excessive ROS and apoptosis-inducing factors, which not only oxidatively damage DNA, protein, lipid, and other biological macromolecules, but also activate c-Jun $\mathrm{N}$-terminal kinase (JNK), P38MAPK, DDR, and P53 pathways, ultimately leading to cell stress apoptosis and senescence ${ }^{21,22,49-51}$. Therefore, accumulation of damaged mitochondria is an important cause of stress apoptosis and aging in $\mathrm{BMSCs}^{52}$. When we further downregulated P53 expression in the BMSCs/Parkin group, mitochondrial translocation of Parkin and mitophagy were increased, significantly reducing damaged mitochondria and ROS in the cells; this was effective against BMSCs stress-induced apoptosis and senescence. Subsequent in vivo studies have also confirmed that upregulating Parkin while downregulating P53 to enhance mitophagy can improve the reparative effect of BMSCs on early steroid-induced ONFH. However, due to limitations imposed by experimental conditions, we could not directly observe survival and differentiation of BMSCs in the osteonecrotic area in vivo, which needs further confirmation by subsequent experiments.

In conclusion, despite the limitations and other deficiencies of this study, we preliminarily confirmed the role and mechanism of P53 and Parkin in regulating mitophagy in BMSCs (Fig. 8). We also proved that upregulating Parkin and downregulating P53 could significantly enhance mitophagy in BMSCs, resist BMSCs stressinduced apoptosis and senescence, and effectively improve the reparative effect of BMSCs on early steroidinduced ONFH.

\section{Methods}

\section{Animals}

All animal studies were approved by the Experimental Animal Bioethics Committee of Guizhou Medical University, Guiyang, China. All animal procedures conformed to the Guide for the Care and Use of Laboratory Animals published by the Directive 2010/63/EU of the European Parliament. We extracted BMSCs from 20 young male New Zealand white rabbits (4-6 weeks old; $1.0-2.0 \mathrm{~kg}$ ) and built ONFH models using 180 adult male New Zealand white rabbits $(4.0-5.0 \mathrm{~kg})$. All rabbits were provided by the Laboratory Animal Center of Guizhou Medical University.

\section{Cell culture}

Our methods for cultivating BMSCs were similar to those in our previous studies ${ }^{53}$. From the young male rabbits, we extracted bilateral distal-femur and proximaltibia bone marrow under sterile conditions and added an appropriate amount of heparin/phosphate-buffered saline (PBS; $1000 \mathrm{U} / \mathrm{mL}$ ) to prevent blood coagulation. We then diluted the bone marrow solution 1:1 with PBS buffer (HyClone, Illinois, USA), blew it into a single-cell suspension, centrifuged it at $600 \mathrm{~g} / \mathrm{min}$ for $5 \mathrm{~min}$, and removed the upper layer of suspended fat. Then the precipitated bone marrow was slowly added to Percoll solution (Pharmacia, New York, USA) dropwise along the wall of the tube to a density of $1.073 \mathrm{~g} / \mathrm{mL}$ and centrifuged at $900 \mathrm{~g} / \mathrm{min}$ for $30 \mathrm{~min}$. We harvested nucleated cells, washed them in PBS buffer, and centrifuged them at $600 \mathrm{~g} / \mathrm{min}$ for $15 \mathrm{~min}$. The cells were resuspended in complete L-glutamine Dulbecco's modified Eagle medium (L-DMEM; GIBCO, Massachusetts, USA) containing 10\% fetal bovine serum (FBS; GIBCO) and $1 \%$ double antibody (HyClone), and cultured at $37^{\circ} \mathrm{C}$ under $5 \% \mathrm{CO}_{2}$. When primary BMSCs had pooled at $80-90 \%$ of the bottom of the flask, we digested the cells with an appropriate amount of $0.25 \%$ trypsin-0.02\% ethylenediaminetetraacetic acid (EDTA; GIBCO) at $37^{\circ} \mathrm{C}$ and passaged them at 1:3. Third-generation BMSCs were used for subsequent experiments.

\section{Osteogenesis induction and identification of BMSCs}

We induced osteogenesis in the BMSCs using the same method as in our previous studies ${ }^{3}$. Cell density of thirdgeneration BMSCs was adjusted to $1 \times 10^{5} / \mathrm{mL}$, and the cells were inoculated on a 6 -well plate with $2 \mathrm{~mL}$ per well. When cell confluence reached $60-70 \%$, we replaced the experimental group with osteogenic-differentiation medium (Cyagen Biosciences, Guangzhou, China) but left the control group in complete L-DMEM. After osteogenic induction for 2 weeks, PBS buffer was sucked out, cells were fixed, calcium nodules were identified by $0.1 \%$ alizarin red staining (Cyagen Biosciences), and ALP activity was detected by a modified Gomori calcium cobalt method (Solarbio, Beijing, China).

\section{Lentivirus infection}

We purchased Lv-P53-EGFP, Lv-shP53-EGFP, and LvParkin-EGFP from Shanghai GeneChem Co., Ltd. (Shanghai, China) A pre-transfection experiment revealed the best multiplicity of infection (MOI; 100) and best transfection conditions (enhanced infection solution ([Eni.s] + Polybrane; both from Shanghai GeneChem). We infected BMSCs with lentivirus and replaced the infected medium with complete L-DMEM $10 \mathrm{~h}$ later. On day 4 after infection, was added L-DMEM containing $2 \mu \mathrm{g} / \mathrm{mL}$ puromycin (Shanghai GeneChem) to screen stable strains. When all cells in the blank control had died, we reduced the concentration of puromycin to $1 \mu \mathrm{g} / \mathrm{mL}$ to maintain screening. 


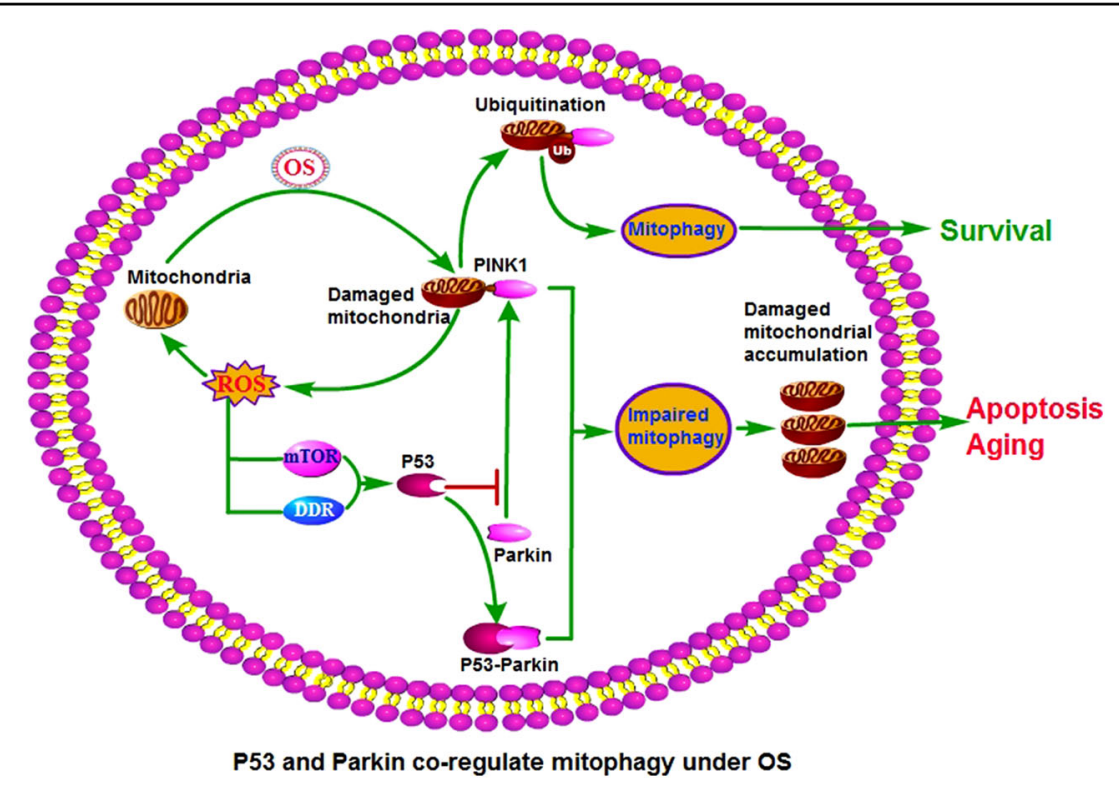

Fig. 8 Hypothetical model of P53 and Parkin co-regulating mitophagy in BMSCs to interfere with cell stress-induced apoptosis and senescence. Under OS, mitochondrial function is impaired and damaged mitochondria release excessive ROS, which can further damage the mitochondria, creating a vicious circle. Moreover, ROS can upregulate P53 expression via the DDR and mTOR pathways. P53 binds to Parkin and inhibits its mitochondrial translocation, the ubiquitination of mitochondrial outer-membrane proteins was inhibited. Eventually, the level of mitophagy decreased, and a large number of mitochondria accumulated in the cells, resulting in apoptosis and senescence of the cells. DDR $=$ DNA damage response; $\mathrm{mTOR}=$ mammalian target of rapamycin.

\section{Real-time PCR (RT-PCR)}

We extracted RNA from BMSCs via column affinity purification (QIAGEN, Hilden, Germany) and synthesized complementary DNAs (cDNAs) using M-MuLV RT Master Mix with Oligo(dT) (Sangon Biotech, Shanghai, China). We performed RT-PCR on a StepOnePlus system (Applied Biosystems, California, USA) in 96-well plates with specific primers and SYBR Green Mix (Sangon Biotech). Rabbit primers (Sangon Biotech) were as follows: Parkin-F: TGACCAGTTGCGTGTGATCTTCG; Parkin-R: GTTGTCTCCTCCAGGCGTGTTG; P53-F: ATGGAGGAG TCGCAGTCGGATC; P53-R: GGTGGT CAGCAGGTTGTTCTCAG; ACTB-F: TCCCTGGAGA AGAGCTACGA; ACTB-R: GTACAGGT CCTTGCGGA TGT. We calculated the fold change value of mRNA expression over that of control using the ${ }^{\Delta \Delta \mathrm{Ct}}$ method.

\section{Isolation of mitochondria and cytoplasm, and extraction of protein}

We isolated mitochondrial and cytoplasmic fractions using a commercial kit (QIAGEN). For whole-cell lysates, cells were lysed with radioimmunoprecipitation assay (RIPA) cell lysate (Beyotime Institute of Biotechnology, Shanghai, China) and centrifuged at $13,000 \mathrm{~g} / \mathrm{min}$ for 10 min to obtain supernatant. All work was performed on wet ice. We quantified proteins using a bicinchoninic acid (BCA) assay (Solarbio) and stored them at $-80^{\circ} \mathrm{C}$.

\section{Immunoprecipitation and immunoblot}

We incubated the cytosolic fractions with antibodies for $12 \mathrm{~h}$ at $4{ }^{\circ} \mathrm{C}$ and then added Protein A/G Plus-Agarose (Sigma-Aldrich, Darmstadt, Germany) for $3 \mathrm{~h}$ at $4{ }^{\circ} \mathrm{C}$ on a rotating device. Immunoprecipitates were collected by centrifugation at $6000 \mathrm{~g} / \mathrm{min}$ at $4{ }^{\circ} \mathrm{C}$ and washed with lysis buffer $(20 \mathrm{mM}$ Tris pH 7.5, $150 \mathrm{mM} \mathrm{NaCl}, 1 \mathrm{mM}$ EDTA, $1 \%$ Triton X-100, proteases, and phosphatase inhibitors; Sigma-Aldrich). We eluted the pellets by heating them at $95^{\circ} \mathrm{C}$ for $5 \mathrm{~min}$ in electrophoresis sample buffer and subjected them to immunoblotting. For immunoblotting, we prepared sodium dodecyl sulfate polyacrylamide gel electrophoresis (SDS-PAGE) gel (Solarbio), added an equal amount of protein for electrophoresis, and transferred the mixture to a polyvinylidene fluoride (PVDF) membrane (Merck Millipore, Darmstadt, Germany). We used rabbit anti-P53 (1:1000; ab154036; Abcam, Cambridge, UK), rabbit anti-Parkin (1:300; BA1682-1; Boster Biological Technology, Ltd., Wuhan, China), rabbit antiVDAC1 (1:1000; ab34726; Abcam), mouse anti-ubiquitin (1:1000; ab52664; Abcam), rabbit anti-NDUFA9 (1:1000; ab128744; Abcam), mouse anti-mitochondrial $70 \mathrm{kDa}$ heat shock protein (mtHsp70; 1:2000; H5147; SigmaAldrich), rabbit anti-P21 (1:1000; bs-10129R; Bioss, Shanghai, China), rabbit anti-P16 (1:1000; A00016-3; Boster), rabbit anti-OPG (1:1500; bs-20624R; Bioss), mouse anti-OCN (1:1000; ab13420; Abcam), rabbit anti- 
Runx2 (1:1000; AV36678; Sigma-Aldrich), and rabbit anti- $\beta$-actin (1:3000; ab8227; Abcam) for primaryantibody response; and horseradish peroxide (HRP)-conjugated goat anti-rabbit and goat anti-mouse immunoglobulin G (IgG; Beyotime) for secondary-antibody response. We used electrochemiluminescence (ECL; Merck Millipore) used for exposure, took photographs using a gel imaging system (Clinx Science Instruments, Ltd., Shanghai, China), and performed quantitative analysis using ImageJ software (1.4.3.67).

\section{Cellular oxidative stress}

In accordance with our previous research ${ }^{35}$, we created a classic OS BMSCs model by treating BMSCs with a high concentration of $\mathrm{H}_{2} \mathrm{O}_{2}(1000 \mu \mathrm{M})$ for $24 \mathrm{~h}$. L-DMEM was used as the control. After P53 knockout and Parkin overexpression in BMSCs per experimental-grouping conditions, we treated the BMSCs in each group with $\mathrm{H}_{2} \mathrm{O}_{2}$ (Chengdu Jinshan Chemical Reagent Co., Ltd., Sichuan, China) for $24 \mathrm{~h}$ and subjected them to OS.

\section{DCFH-DA staining}

After we washed the cells three times in PBS buffer, we prepared the reaction mixture reagent using a $2^{\prime}, 7^{\prime}$ Dichlorodihydrofluorescein diacetate (DCFH-DA) fluorescent-probe kit (Sigma-Aldrich) per manufacturer's instructions. Cells were incubated at $37^{\circ} \mathrm{C}$ for $30 \mathrm{~min}$. We observed green fluorescence under a confocal microscope (Zeiss, Oberkochen, Germany) in the fluorescein isothiocyanate (FITC) green-fluorescence channel.

\section{JC-1 staining}

We washed the cells three times in PBS buffer and prepared a JC-1 fluorescent probe using a MMP detection kit (Nanjiang KeyGen Biotech Co., Ltd, Jiangsu, China) per manufacturer's instructions. After staining at $37^{\circ} \mathrm{C}$ for $30 \mathrm{~min}$, we observed red and green fluorescence under a confocal microscope (Zeiss).

\section{MitoTracker Green staining}

We washed the cells three times in PBS buffer and prepared MitoTracker Green stock solution and working solution using a MitoTracker Green fluorescent probe kit (Beyotime) per manufacturer's instructions. Cells were incubated with the working solution at $37^{\circ} \mathrm{C}$ for $30 \mathrm{~min}$. We observed green fluorescence in the FITC greenfluorescence channel using a laser confocal microscope (Zeiss).

\section{$\beta$-gal staining}

We fixed the cells at room temperature (RT) for $15 \mathrm{~min}$ and prepared a staining working solution using a cell senescence $\beta$-gal staining kit (Beyotime) per manufacturer's instructions. After the working solution was added, the cells were incubated at $37^{\circ} \mathrm{C}$ overnight and observed using a general optical microscope.

\section{TUNEL-DAPI staining}

We fixed the cells in $4 \%$ paraformaldehyde for $30 \mathrm{~min}$, permeabilized them with $0.3 \%$ Triton X-100 for 5 min at RT, and prepared a TUNEL assay solution using a TUNEL Apoptosis Detection Kit (Beyotime) per manufacturer's instructions. The assay solution and cells were incubated at $37^{\circ} \mathrm{C}$ for $60 \mathrm{~min}$ in the dark and stained with $20 \mu \mathrm{g} / \mathrm{mL}$ DAPI (Solarbio) for $3 \mathrm{~min}$. We then observed red and blue fluorescence using a laser confocal microscope (Zeiss).

\section{Annexin V-FITC/PI detection of apoptosis}

Using an Annexin V-FITC Apoptosis Detection Kit (BD Biosciences, San Jose, California, USA) per manufacturer's instructions, we directly added $5 \mu \mathrm{L}$ Annexin $\mathrm{V}$-FITC and $5 \mu \mathrm{L} \mathrm{PI}$, gently vortexed the cells, and incubated them at RT for $15 \mathrm{~min}$ in the dark. We then detected apoptosis in the cells via flow cytometry (Beckman Coulter, Indianapolis, Indiana, USA).

\section{TEM observation of mitophagy}

We trypsinized the cells, collected the cell pellet, added $2.5 \%$ glutaraldehyde solution, and fixed the cells at $4{ }^{\circ} \mathrm{C}$ overnight. After dehydration, embedding, ultra-thin sectioning, uranium dyeing, and lead staining, we observed mitophagy using a TEM (Hitachi, Tokyo, Japan).

\section{XACB}

We removed cortical bone and cartilage from fresh porcine vertebrae (Laboratory Animal Center of Guizhou Medical University, Guizhou, China) according to the procedures established by $\mathrm{Li}$ et al. and Long et al. ${ }^{54-56}$. Cancellous-bone blocks were shaped into cylinders $6 \mathrm{~mm}$ in diameter at the bottom and $20 \mathrm{~mm}$ high using a cutting and molding device. After repeated cleanings with distilled water, we subjected the cylinders to $30 \% \mathrm{H}_{2} \mathrm{O}_{2}$ deproteinization for $48 \mathrm{~h}, 1: 1$ chloroform/methanol degreasing for $24 \mathrm{~h}$, freeze-drying in a $-50^{\circ} \mathrm{C}$ freeze dryer for $24 \mathrm{~h}$, ethylene oxide closed disinfection for $24 \mathrm{~h}$ to obtain sterile XACB.

\section{Detection of biocompatibility between BMSCs and XACB}

Based on our previous research ${ }^{3}$, we cut XACB into small cylinders $5 \mathrm{~mm}$ in diameter at the bottom and $5 \mathrm{~mm}$ high and immersed them in complete L-DMEM for $24 \mathrm{~h}$. We added BMSCs suspension $\left(1 \times 10^{7} / \mathrm{mL}\right)$ to $\mathrm{XACB}$, allowed the cells to adhere for $3 \mathrm{~h}$ in an incubator $\left(37^{\circ} \mathrm{C}\right.$, $5 \% \mathrm{CO}_{2}$ ), slowly added complete L-DMEM along the wall of the culture plate, and continued to culture the mixture in the incubator $\left(37^{\circ} \mathrm{C}, 5 \% \mathrm{CO}_{2}\right)$ to construct tissueengineered bone (BMSCs/XACB). Each day for the next 
week, we took five pieces of BMSCs/XACB out of the incubator, collected cells from them via digestion and centrifugation, and added $10 \mu \mathrm{L}$ of Cell Counting Kit-8 (CCK-8) solution (Solarbio) to each well. We measured the absorbance value $(450 \mathrm{~nm})$ until day 7 , when we drew the growth curve based on culture time and absorbance value. We cultured XACB and BMSCs on day 6 and observed the growth of BMSCs on the surface of XACB using a SEM (Hitachi).

\section{Early steroid-induced ONFH rabbit model}

After weighing and disinfection, we injected LPS $(10 \mu \mathrm{g} /$ $\mathrm{kg} / \mathrm{d}$; Sigma-Aldrich) into the ear veins of 180 adult male rabbits for 2 days. On day 2, after LPS injection, we injected methylprednisolone $(40 \mathrm{mg} / \mathrm{kg} / \mathrm{d}$; Pfizer) into the rabbits' gluteal muscles for 3 days, weighed before each injection ${ }^{57-60}$. Rabbits in the control group were injected with saline. Eight weeks after modeling, we stained femoral-head tissue with $\mathrm{H} \& \mathrm{E}$, and the successful model was used for in vivo experiments.

\section{Transplantation of tissue-engineered bone}

Rabbits were shaven and their skin cleaned 1 week before surgery. We injected penicillin $(50,000 \mathrm{U} / \mathrm{kg}$; CSPC Pharmaceutical Group, Ltd, Hebei, China) intramuscularly $30 \mathrm{~min}$ before surgery to prevent infection. We anesthetized the rabbits with $3 \%$ pentobarbital $(1 \mathrm{~mL} / \mathrm{kg}$; Merck Millipore) by ear vein, put them into a prone position, and disinfected the surgical site. A posterior lateral-arc incision of about $5 \mathrm{~cm}$ was made to the right hip joint; after exposing this joint, we exposed the femoral head using a T-shaped incision. A 3-mm diameter spherical grinding drill was used at the junction of bone and cartilage, drilling from the posterolateral side to the anteromedial side of the femoral head at a depth of 4-6 $\mathrm{mm}$ (the diameter of the femoral head in adult rabbits is $6-8 \mathrm{~mm}$ ); and then lesion debridement (LD) was performed with a curette. We implanted BMSCs/XACB according to experimental grouping: control (LD), XACB, XACB/BMSCs, XACB/BMSCs/ Parkin, XACB/BMSCs/shP53, or XACB/BMSCs/Parkin/ shP53. A gelatin sponge (Baotou Dongbao Biotechnology Co., Ltd, Shenzhen, China) was then inserted into the tunnel. Operations were performed on the right side, and the left side was the self-control. Penicillin $(50,000 \mathrm{U} / \mathrm{kg})$ was used continuously for 3 days after the operation to prevent infection. Randomization was used to determine animals were allocated to experimental groups. The operator or investigator was blinded to the grouping.

\section{X-ray examination}

At 12 weeks after surgery, after intravenous anesthesia with $3 \%$ pentobarbital sodium $(1 \mathrm{~mL} / \mathrm{kg})$, we examined all experimental animals by X-ray $(60 \mathrm{kV}, 50 \mathrm{~mA}, 0.15 \mathrm{~s})$, with the animals in the prone position.

\section{Observation of the femoral-head specimen}

At 12 weeks after surgery, we removed the femoral head, observed its surface, dissected it longitudinally from the middle, and observed repair of the femoral-head defect.

\section{H\&E and Masson staining}

At 12 weeks after surgery, bone tissue was routinely decalcified, dehydrated, and paraffin-embedded to make $3-\mu \mathrm{m}$-thick sections, which we stained using a H\&E staining kit (Solarbio) per manufacturer's instructions. Paraffin sections were dewaxed to water and stained, made transparent, mounted, and microscopically examined using a Masson's trichrome staining kit (Solarbio) per manufacturer's instructions.

\section{Extraction of total protein from bone tissue}

We took out the femoral head and removed the normal bone and cartilage tissue on its surface with a rongeur; the remaining bone tissue was washed with PBS. We crushed the bone tissue using a hammer tissue pulverizer and then quickly transferred the fragments into a mortar containing liquid nitrogen. The bone tissue was ground into a powder (with adequate liquid nitrogen maintained during grinding), the powdered bone tissue was collected into an Eppendorf (EP) tube, and a protein lysate was added to each tube $(200 \mu \mathrm{L}$ lysate per $100 \mathrm{mg}$ bone tissue). After cleavage on ice for $30 \mathrm{~min}$, we pipetted the lysate from the $E P$ tube into a second EP tube, centrifuged it at $12,000 \mathrm{rpm}$ for $15 \mathrm{~min}$ at $4{ }^{\circ} \mathrm{C}$, and harvested the supernatant for western blot analysis.

\section{Statistical analysis}

All statistical data were calculated and graphed using GraphPad Prism software version 6 (GraphPad Software, San Diego, California, USA). To assess statistical significance, we used a Kolmogorov-Smirnov test to analyze normal distribution of data, a two-tailed unpaired Student's $t$ test for analyses involving only two groups for comparison, and analysis of variance (ANOVA) and appropriate post hoc tests for analyses involving $>2$ groups for comparisons. All error bars are expressed as mean \pm standard deviation (SD). $P<0.05$ was considered statistically significant.

\footnotetext{
Acknowledgements

This work was supported by the National Natural Science Foundation of China (Grant Nos. 81902226 and 81860387), the Science and Technology Fund of Guizhou Provincial Department of Health (Grant Nos. gzwjkj2019-1-135 and gzwjkj2018-1-008), the Guizhou Provincial Science and Technology Department-Guizhou Medical University Joint Fund (Grant No. [2017]7197), the Guiyang Science and Technology Bureau-Guizhou Medical University Joint Fund (Grant No. [2017]5-2), and the Special Project of Academic New-seedling
} 
Cultivation and Innovation Exploration of Guizhou Medical University in 2018 (Qiankehe Platform Talents Grant No. [2018]5779-43).

\section{Data availability}

The additional data or reagents are available from the corresponding author upon reasonable request.

\section{Conflict of interest}

The authors declare that they have no conflict of interest.

\section{Publisher's note}

Springer Nature remains neutral with regard to jurisdictional claims in published maps and institutional affiliations.

Supplementary Information accompanies this paper at (https://doi.org/ 10.1038/s41419-020-2238-1).

Received: 19 August 2019 Revised: 20 December 2019 Accepted: 20 December 2019

Published online: 20 January 2020

\section{References}

1. Peng, W. X. \& Wang, L. Adenovirus-mediated expression of BMP-2 and BFGF in bone marrow mesenchymal stem cells combined with demineralized bone matrix for repair of femoral head osteonecrosis in beagle dogs. Cell Physiol. Biochem. 43, 1648-1662 (2017).

2. Liao, $\mathrm{H}$. et al. Bone mesenchymal stem cells co-expressing VEGF and BMP-6 genes to combat avascular necrosis of the femoral head. Exp. Ther. Med. 15, 954-962 (2018).

3. Zhang, F. et al. Role of FGF-2 transfected bone marrow mesenchymal stem cells in engineered bone tissue for repair of avascular necrosis of femoral head in rabbits. Cell Physiol. Biochem. 48, 773-784 (2018).

4. Fan, L. et al. Transplantation of hypoxia preconditioned bone marrow mesenchymal stem cells enhances angiogenesis and osteogenesis in rabbit femoral head osteonecrosis. Bone 81, 544-553 (2015).

5. Xu, J. et al. High density lipoprotein protects mesenchymal stem cells from oxidative stress-induced apoptosis via activation of the PI3K/Akt pathway and suppression of reactive oxygen species. Int. J. Mol. Sci. 13, 17104e20 (2012).

6. Wang, $X$. et al. Hsp20-engineered mesenchymal stem cells are resistant to oxidative stress via enhanced activation of Akt and increased secretion of growth factors. Stem Cells 27, 3021 e31 (2009).

7. Pasha, Z. et al. Preconditioning enhances cell survival and differentiation of stem cells during transplantation in infarcted myocardium. Cardiovasc. Res. 77, $134 \mathrm{e} 42$ (2008)

8. Hu, X. F., Wang, L., Xiang, G., Lei, W. \& Feng, Y. F. Angiogenesis impairment by the NADPH oxidase-triggered oxidative stress at the bone-implant interface: critical mechanisms and therapeutic targets for implant failure under hyperglycemic conditions in diabetes. Acta Biomater. 73, 470-487 (2018).

9. McGarry, T., Biniecka, M., Veale, D. J. \& Fearon, U. Hypoxia, oxidative stress and inflammation. Free Radic. Biol. Med. 125, 15-24 (2018).

10. Wauquier, F., Leotoing, L., Coxam, V., Guicheux, J. \& Wittrant, Y. Oxidative stress in bone remodelling and disease. Trends Mol. Med. 15, 468-477 (2009).

11. Deng, G. et al. Treatment of steroid-induced osteonecrosis of the femoral head using porous Se@SiO2 nanocomposites to suppress reactive oxygen species. Sci. Rep. 7, 43914 (2017).

12. Li, R. et al. Stem cell therapy for treating osteonecrosis of the femoral head: From clinical applications to related basic research. Stem Cell Res. Ther. 9, 291 (2018).

13. Sung, Y. J. et al. Mitochondrial Lon sequesters and stabilizes p53 in the matrix to restrain apoptosis under oxidative stress via its chaperone activity. Cell Death Dis. 9, 697 (2018).

14. $\mathrm{Yu}, \mathrm{H}$. H. et al. Stable overexpression of DJ-1 protects H9c2 cells against oxidative stress under a hypoxia condition. Cell Biochem. Funct. 31, 643-651 (2013).

15. Wei, H., Li, Z., Hu, S., Chen, X. \& Cong, X. Apoptosis of mesenchymal stem cells induced by hydrogen peroxide concerns both endoplasmic reticulum stress and mitochondrial death pathway through regulation of caspases, p38 and jnk. J. Cell Biochem. 111, 967-978 (2010).

16. Di Rita, A. et al. HUWE1 E3 ligase promotes PINK1/PARKIN-independent mitophagy by regulating AMBRA1 activation via IKKa. Nat. Commun. 9, 3755 (2018).

17. Wang, $L$. et al. PTEN-L is a novel protein phosphatase for ubiquitin dephosphorylation to inhibit PINK1-Parkin-mediated mitophagy. Cell Res. 28, 787-802 (2018).

18. Tang, C. et al. Activation of BNIP3-mediated mitophagy protects against renal ischemia- reperfusion injury. Cell Death Dis. 10, 677 (2019).

19. Wang, D. B., Kinoshita, C., Kinoshita, Y. \& Morrison, R. S. p53 and mitochondrial function in neurons. Biochim Biophys. Acta 1842, 1186-1197 (2014).

20. Korolchuk, V. I., Miwa, S., Carroll, B. \& von Zglinicki, T. Mitochondria in cell senescence: is mitophagy the weakest link? EBioMedicine 21, 7-13 (2017).

21. Tian, Z. et al. Role of mitophagy regulation by ROS in hepatic stellate cells during acute liver failure. Am. J. Physiol. Gastrointest. Liver Physiol. 315, G374-G384 (2018).

22. Correia-Melo, C. et al. Mitochondria are required for pro-ageing features of the senescent phenotype. EMBO J. 35, 724-742 (2016).

23. Vincow, E. S. et al. The PINK1-Parkin pathway promotes both mitophagy and selective respiratory chain turnover in vivo. Proc. Natl Acad. Sci. USA 110 6400-6405 (2013).

24. Whitworth, A. J. \& Pallanck, L. J. PINK1/Parkin mitophagy and neurodegeneration-what do we really know in vivo? Curr. Opin. Genet Dev. $\mathbf{4 4}$ 47-53 (2017).

25. Childs, B. G., Durik, M., Baker, D. J. \& van Deursen, J. M. Cellular senescence in aging and age-related disease: from mechanisms to therapy. Nat. Med. 21, 1424-1435 (2015).

26. Nacarelli, T., Azar, A. \& Sell, C. Aberrant mTOR activation in senescence and aging: a mitochondrial stress response? Exp. Gerontol. 68, 66-70 (2015).

27. Lai, K. P. et al. S6K1 is a multifaceted regulator of Mdm2 that connects nutrient status and DNA damage response. EMBO J. 29, 2994-3006 (2010).

28. Jung, S. H. et al. mTOR kinase leads to PTEN-loss-induced cellular senescence by phosphorylating p53. Oncogene 38, 1639-1650 (2019).

29. Jung, Y. Y. et al. Loss of Parkin reduces inflammatory arthritis by inhibiting p53 degradation. Redox Biol. 12, 666-673 (2017).

30. Hoshino, A. et al. Cytosolic p53 inhibits Parkin-mediated mitophagy and promotes mitochondrial dysfunction in the mouse heart. Nat. Commun. 4, 2308 (2013).

31. Manzella, N. et al. Monoamine oxidase-A is a novel driver of stress-induced premature senescence through inhibition of parkin-mediated mitophagy. Aging Cell 17, e12811 (2018)

32. Pittenger, M. F. et al. Multilineage potential of adult human mesenchymal stem cells. Science 284, 143-147 (1999).

33. Jiang, $Y$. et al. Pluripotency of mesenchymal stem cells derived from adult marrow. Nature 418, 41-49 (2002).

34. Shen, W. C. et al. Methylation and PTEN activation in dental pulp mesenchymal stem cells promotes osteogenesis and reduces oncogenesis. Nat. Commun. 10, 2226 (2019)

35. Zhang, F. et al. New strategy of bone marrow mesenchymal stem cells against oxidative stress injury via Nrf2 pathway: oxidative stress preconditioning. J. Cell Biochem 120, 19902-19914 (2019).

36. Abate, M. et al. Mitochondria as playmakers of apoptosis, autophagy and senescence. Semin Cell Dev. Biol. 18, S1084-S9521 (2019).

37. Lin, Q. et al. PINK1-parkin pathway of mitophagy protects against contrastinduced acute kidney injury via decreasing mitochondrial ROS and NLRP3 inflammasome activation. Redox Biol. 26, 101254 (2019).

38. Guo, T. et al. Sonodynamic therapy inhibits palmitate-induced beta cell dysfunction via PINK1/Parkin-dependent mitophagy. Cell Death Dis. 10, 457 (2019).

39. Miller, S. \& Muqit, M. M. K. Therapeutic approaches to enhance PINK1/Parkin mediated mitophagy for the treatment of Parkinson's disease. Neurosci. Lett. 705, 7-13 (2019).

40. Kokubun, T., Saitoh, S. I., Miura, S., Ishida, T. \& Takeishi, Y. Telomerase plays a pivotal role in collateral growth under ischemia by suppressing age-induced oxidative stress, expression of p53, and pro-apoptotic proteins. Int. Heart J. 60, 736-745 (2019).

41. Liu, T. et al. SIRT1 reverses senescence via enhancing autophagy and attenuates oxidative stress-induced apoptosis through promoting p53 degradation. Int J. Biol. Macromol. 117, 225-234 (2018). 
42. Wang, $Y$. et al. PINK1/Parkin-mediated mitophagy is activated in cisplatin nephrotoxicity to protect against kidney injury. Cell Death Dis. 9, 1113 (2018).

43. Nguyen, V. T. et al. Does the harvesting site influence the osteogenic potential of mesenchymal stem cells? Stem Cells Int. 2019, 9178436 (2019).

44. $\mathrm{Xu}, \mathrm{H}$. J. et al. Down-regulation of exosomal microRNA-224-3p derived from bone marrow-derived mesenchymal stem cells potentiates angiogenesis in traumatic osteonecrosis of the femoral head. FASEB $\mathrm{J}$. 33, 8055-8068 (2019).

45. Wang, B., Yu, P., Li, T., Bian, Y. \& Weng, X. MicroRNA expression in bone marrow mesenchymal stem cells from mice with steroid-induced osteonecrosis of the femoral head. Mol. Med. Rep. 12, 7447-7454 (2015).

46. Ferro, F. et al. Survival/adaptation of bone marrow-derived mesenchymal stem cells after long-term starvation through selective processes. Stem Cells $\mathbf{3 7}$, 813-827 (2019).

47. Volkmer, E. et al. Hypoxic preconditioning of human mesenchymal stem cells overcomes hypoxia-induced inhibition of osteogenic differentiation. Tissue Eng. Part A 16, 153-164 (2010).

48. Gong, G. et al. Parkin-mediated mitophagy directs perinatal cardiac metabolic maturation in mice. Science 350, aad2459 (2015).

49. Bu, H., Wedel, S., Cavinato, M. \& Jansen-Dürr, P. MicroRNA regulation of oxidative stress-induced cellular senescence. Oxid. Med. Cell Longev. 2017, 2398696 (2017).

50. Liguori, I. et al. Oxidative stress, aging, and diseases. Clin. Inter. Aging 13, 757-772 (2018)

51. Oh, J., Lee, Y. D. \& Wagers, A. J. Stem cell aging: mechanisms, regulators and therapeutic opportunities. Nat. Med. 20, 870-880 (2014).
52. Ashrafi, G. \& Schwarz, T. L. The pathways of mitophagy for quality control and clearance of mitochondria. Cell Death Differ. 20, 31-42 (2013).

53. Peng, W. et al. Effects of lentiviral transfection containing bFGF gene on the biological characteristics of rabbit BMSCs. J. Cell Biochem. 119, 8389-8397 (2018).

54. Li, Z. J., Lu, C. T. \& Lai, R. F. Ectopic osteogenesis effect of antigen-extracted xenogeneic cancellous bone graft with chitosan/rhBMP-2/bFGF sequential sustained-release nanocapsules. J. Biomater. Appl. 33, 23-43 (2018).

55. Li, Z. J. et al. Antigen-extracted xenogeneic cancellous bone graft with recombinant human bone morphogenetic protein-2 enhances bone regeneration in repair of mandibular defect in rabbits. Kaohsiung J. Med Sci. 31 18-25 (2015).

56. Long, B. et al. Evaluation of a novel reconstituted bone xenograft using processed bovine cancellous bone in combination with purified bovine bone morphogenetic protein. Xenotransplantation 19, 122-132 (2012).

57. Wang, T. et al. Role of mesenchymal stem cells on differentiation in steroidinduced avascular necrosis of the femoral head. Exp. Ther. Med. 13, 669-675 (2017).

58. Matsui, M. et al. Experimental steroid-induced osteonecrosis in adult rabbits with hypersensitivity vasculitiy. Clin. Orthop. Relat. Res. 277, 61-72 (1992).

59. Yamamoto, T., Hirano, K., Tsutsui, H., Sugioka, Y. \& Sueishi, K. Corticosteroid enhances the experimental induction of osteonecrosis in rabbits with Shwartzman reaction. Clin. Orthop. Relat. Res. 316, 235-243 (1995).

60. Ryoo, S. et al. Effect of lipopolysaccharide (LPS) on mouse model of steroidinduced avascular necrosis in the femoral head (ANFH). J. Microbiol. Biotechnol. 24, 394-400 (2014) 